\title{
Criblage De Quelques Genotypes De Ble Dur (Triticum Durum Desf.) Sous Un Stress Salin En Culture De Pot
}

\author{
Sourour Ayed, PhD \\ Afef Othmani, PhD
}

University of Carthage, Field Crop Laboratory, Regional Research Development Office of Agriculture in Semi Arid North West of Kef, Tunisia

\section{Sadreddine Beji, PhD}

Higher Agriculture School of Kef, Boulifa, Kef, Tunisia

\section{Hajer Amara, Pr}

Genetic and Plant Breeding Laboratory, Department of Agronomy and Biotechnology. National Agronomic Institute of Tunisia

\section{doi: 10.19044/esj.2016.v12n9p313～URL:http://dx.doi.org/10.19044/esj.2016.v12n9p313}

\begin{abstract}
In this study, five durum wheat (Triticum turgidum ssp durum) genotypes: Agili, Arbi, Derbassi, Karim and Maali were assessed for 04 agronomical parameters associated with salinity-tolerance. Genotypes were grown under glasshouse conditions and exposed to five salinity levels (control, $3 \mathrm{~g} / \mathrm{l}, 6 \mathrm{~g} / \mathrm{l}, 9 \mathrm{~g} / \mathrm{l}$ et $12 \mathrm{~g} / \mathrm{l}$ of $\mathrm{NaCl}$ ) to evaluate their tolerance using their biomass and the relative salt-susceptibility index: R.S.S.I = R.B.D./S.I.I. (R.B.D.: relative biomass deficit and S.I.I.: salinity intensity index).Genotypic screening was also based on agro-morphological traits: tillers number/plant, plant height, grains number/spike and 1000 kernel weight. Analysis of variance revealed a significant $(\mathrm{p}<0.001)$ difference between treatments and genotypes. This difference indicated a large variability between genotypes. Results showed also that Maali variety had the higher agronomic performance and the lower R.S.S.I than other genotypes.
\end{abstract}

Keywords: Genotypes, salinity-tolerance

Résumé

Ce travail a porté sur l'évaluation du comportement de cinq génotypes de blé dur (Triticum turgidum ssp durum) à savoir Agili, Arbi, Derbassi, Karim et Maali soumis à différents niveaux de salinité $(0,3,6,9$ et 
$12 \mathrm{~g} / \mathrm{l})$. Les essais ont été effectués en pot sous serre à l'Ecole Supérieure d'Agriculture du Kef. L'évaluation de la tolérance au stress salin a été réalisé au moyen de leur biomasse et de leur indice de sensibilité relative au sel: I.S.R.S. = D.R.B./I.I.S. (D.R.B.: déficit relatif de biomasse et I.I.S.: indice d'intensité de la salinité). Le criblage des génotypes a été basé également sur un ensemble de paramètres agronomiques: nombre de talles par plante, hauteur de la végétation, nombre de grains par épi et poids de mille grains. L'analyse de la variance a montré une différence significative $(p<0,001)$ entre les traitements et entre les génotypes pour tous les paramètres étudiés, indiquant une large variabilité entre les génotypes. Les résultats obtenus révèlent que la variété Maali s'est distinguée par une tolérance à la salinité en maintenant un nombre de grains/épi et un poids de mille grains importants comparativement au témoin non stressé et montrant des I.I.S les plus faibles en comparaison avec les autres génotypes.

Mots clés: Blé dur, indice de sensibilité relative au sel, paramètres agronomiques, stress salin

\section{Introduction}

Les stress environnementaux en l'occurrence le stress salin entrave la production agricole dans toutes les zones et plus particulièrement les zones arides et semi arides où la salinisation s'impose de plus en plus fortement étant donné la rareté des ressources en eau (conditions climatiques) et la médiocrité de leur qualité (saumâtres) (Levigneron et al., 1995). La Tunisie est concernée par ce problème vue l'importante partie de ses sols affectés par le sel (10\% de superficie totale du pays) ainsi que la qualité d'eau médiocre employée dans l'irrigation, la salinité affecte environ le tiers des superficies irrigués (FAO, 2013). L'impact néfaste de la salinité se traduit par la conjonction d'un effet indirect sur le potentiel hydrique se traduisant par une réduction de la disponibilité de l'eau pour la plante (Niu et al., 1995; Al Karaki, 2000). La salinité affecte presque la totalité des processus de développement des plantes, la germination, la croissance des plantules, la phase végétative et la floraison à des degrés variables (Delgado et al., 1994; Cordovilla et al., 1995). Puisque la salinité, facteur important parmi les contraintes à l'intensification de la culture du blé dur, mérite dès lors une attention particulière. La sélection de variétés de blé dur adaptées à l'irrigation par de l'eau chargée est donc une nécessité primordiale (Munns et Tester, 2008). L'intérêt porté aux caractères quantitatifs d'adaptation à la contrainte saline a suscité l'attention de nombreux chercheurs. Dans ce sens, plusieurs critères de tolérance agronomiques (le rendement, la vigueur, les dommages foliaires et la hauteur de la végétation), vus leur taux de héritabilité élevé, ont été identifiés dans le but de la discrimination entre les 
différents génotypes pour la tolérance à ce stress (Shannon, 1997; Mainassara et al., 2009; Gholamin et al., 2010). D'autres indicateurs de tolérance ont été reportés, notamment les critères physiologiques comme l'accumulation d'ions, taux de sucres solubles et de la proline. Chez le blé, plusieurs travaux de recherche ont été focalisés sur l'étude de l'effet de la salinité (Cheraghi, 2004; Dehdari et al., 2007, Asgaria et al., 2012). Ces auteurs ont rapporté une corrélation étroite entre les rendements en conditions témoin et sous stress salin. Dans ce cadre s'intègre ce travail de recherche qui vise à étudier le comportement de quelques génotypes de blé en se basant sur quelques caractéristiques agro-morphologiques associées à la tolérance au sel et pouvant servir comme de critères simples de sélection chez le blé dur vis-à-vis du stress salin afin de sélectionner les meilleurs génotypes tolérants.

\section{Matériel et Méthodes \\ Matériel végétal}

Ce travail a porté sur l'évaluation de 05 génotypes de blé dur (Triticum durum Desf.) à savoir Agili, Arbi, Derbassi, Karim et Maali vis-àvis du stress salin. Ces génotypes sont choisis parmi 10 génotypes précédemment évalués pour leur tolérance au stress salin au stade juvénile par Ayed et al. (2014).

\section{Culture en pot}

Les grains ont été semés dans des pots de $30 \mathrm{~cm}$ de diamètre remplie d'un mélange de $1 / 3$ tourbe et $2 / 3$ sable à raison de 10 grains/ pot. L'essai est installé dans une serre contrôlée à l'Ecole Supérieure d'Agriculture du Kef, selon un dispositif complètement randomisé à raison de 3 répétitions pour chaque dose et pour chaque génotype.

\section{Application du stress salin}

Les différentes concentrations de $\mathrm{NaCl}$ ont été portées dans l'eau d'irrigation. Les irrigations ont été réalisées avec une fréquence moyenne de 1 fois tous les trois jours. Cinq traitements ont été testés:

Traitement $1: 0 \mathrm{~g} / 1$ de $\mathrm{NaCl}$ (Témoin)

Traitement $2: 3 \mathrm{~g} / \mathrm{l}$ de $\mathrm{NaCl}$

Traitement $3: 6 \mathrm{~g} / \mathrm{l}$ de $\mathrm{NaCl}$

Traitement $4: 9 \mathrm{~g} / \mathrm{l}$ de $\mathrm{NaCl}$

Traitement $5: 12 \mathrm{~g} / \mathrm{l}$ de $\mathrm{NaCl}$ 


\section{Paramètres mesurés}

L'évaluation de la réponse des génotypes a été basée sur quatre paramètres à savoir le nombre de talles par plante, la hauteur, le nombre de grains par épi et le poids de mille grains.

La réponse à la salinité de ces génotypes a été déterminé également par la mesure de l'indice de sensibilité relative au sel ou I.S.R.S. qui traduit le rapport de la sensibilité d'un cultivar donné (exprimée par le déficit relatif de biomasse dû au sel ou D.R.B.) à la sensibilité moyenne de l'ensemble des cultivars ou indice d'intensité de la salinité (I.I.S.). Il est calculé comme suit:

I.S.R.S= D.R.B/I.I.S (Fisher et al., 1978) étant donné D.R.B $=\left(\mathrm{BT}_{\mathrm{t}}-\right.$ $\left.\mathrm{BT}_{\mathrm{s}}\right) / \mathrm{BT}_{\mathrm{t}}$ et I.I.S $=\left(\mathrm{M}_{\mathrm{t}}-\mathrm{M}_{\mathrm{s}}\right) / \mathrm{M}_{\mathrm{t}}$ avec BT: biomasse totale moyenne du génotype en absence de sel (t) ou sous stress salin (s), M: biomasse totale moyenne de tous les génotypes en absence de sel (t) ou sous stress salin (s).

\section{Analyses statistiques}

L'ensemble des données obtenus ont fait l'objet d'une analyse de la variance à un et à deux facteurs fixes de classification. Chaque fois que l'effet de l'un de ces facteurs ou leurs interactions se sont montrés significatifs, nous avons procédé à une comparaison des moyennes selon le test du Duncan au seuil de 5\% et 1\%. L'ensemble des analyses statistiques a été effectué avec le logiciel SAS version 9.2.

\section{Résultats et Discussion}

\section{Effet de la salinité sur les paramètres agro-morphologiques}

Les génotypes ont fait l'objet d'une évaluation agro- morphologiques en mesurant la hauteur de la végétation, le nombre de talles par plante, le nombre de grains par épi et le poids de 1000 grains afin de cribler les génotypes les plus tolérants à la salinité. L'analyse de la variance révèle un effet hautement significatif de différents niveaux de salinité pour tous les paramètres et sur l'ensemble des génotypes. La différence entre les génotypes est hautement significative pour la plupart des paramètres étudiés (tableau 1). Des interactions traitement $\mathrm{x}$ génotypes hautement significatives ont été noté ce qui indique que les génotypes étudiés ont des comportements différents vis-à-vis du stress salin et se caractérisent par une variabilité génotypique importante. Ces résultats sont en concordance avec ceux obtenus par Hemati and Pakniyat (2006). 
Tableau 1: Analyse de la variance (test $\mathrm{F}$ ) relative aux paramètres mesurés chez les différents génotypes de blé dur

\begin{tabular}{|c|c|c|c|c|c|}
\hline $\begin{array}{c}\text { Sources de } \\
\text { variation }\end{array}$ & ddl & $\begin{array}{c}\text { Hauteur de la } \\
\text { végétation }\end{array}$ & $\begin{array}{c}\text { Nombre de } \\
\text { talles/ plante }\end{array}$ & $\begin{array}{c}\text { Nombre } \\
\text { de grains } \\
\text { /épi }\end{array}$ & $\begin{array}{c}\text { Poids de } \\
\mathbf{1 0 0 0} \text { grains }\end{array}$ \\
\hline Genotype & 4 & $164.45^{* *}$ & $23.41^{* *}$ & $141.40^{* *}$ & $42.83^{* *}$ \\
\hline Traitement & 4 & $277.00^{* *}$ & $33.63^{* *}$ & $121.15^{* *}$ & $154.35^{* *}$ \\
\hline $\begin{array}{c}\text { Genotype } \mathrm{x} \\
\text { traitement }\end{array}$ & 16 & $10.90^{* *}$ & $5.96^{* *}$ & $11.46^{* *}$ & $6.64^{* *}$ \\
\hline
\end{tabular}

ddl: degré de liberté ; ns: test non significatif $(\mathrm{p}>0.05)$, *: significatif $(0.01<\mathrm{p}<0.05$, $\mathrm{P}<0.01) ; * *$ : hautement significatif $(\mathrm{P}<0.001)$.

\section{La hauteur de la végétation}

L'analyse de la variance des génotypes testés a montré une distinction claire entre les variétés améliorées et les variétés autochtones. En effet, ces dernières se sont caractérisées par une paille haute quelque soit le niveau de salinité. Ceci est largement documenté et est dû au fait que les génotypes améliorés contiennent des gènes de nanisme (Deghaïs et al., 2007). Tous les génotypes ont montré une réduction de la hauteur suite à l'irrigation par l'eau saline (figure 1). Cependant, les génotypes Arbi, Derbassi et Agili sont les plus affectés par la salinité. Plusieurs auteurs ont noté que la réduction de l'accroissement des tiges serait une stratégie d'adaptation à la contrainte saline plus qu'un simple effet de la salinité (Ben Naceur, 2001). Cela peut expliquer que les variétés autochtones pourraient être plus adaptées aux conditions de stress salin. Selon Ben Naceur et al. (2001) et Hameed et al. (2008), l'effet de la salinité se traduit généralement par une réduction de la croissance végétative. Katerji et al. (2006) ont rapporté que la hauteur de la végétation est un paramètre indicateur de l'effet inhibiteur du sel sur la croissance des plantes. En effet, ce stress retarde la croissance des pousses qui sont plus sensibles au sel que les racines (Läuchli et Epstein, 1990) et cette baisse peut être expliquée par un raccourcissement des entre nœuds. En fait, l'effet du stress osmotique induit par le sel agit sur l'élongation des cellules d'une part (Fricke et Peters, 2002) et sur la nutrition minérale d'autre part (Munns, 2002; Parida et Das, 2005). Selon ces auteurs, le stress réduit la vitesse d'élongation des cellules de la tige, en partie, à cause de l'accumulation de l'acide abscissique. 


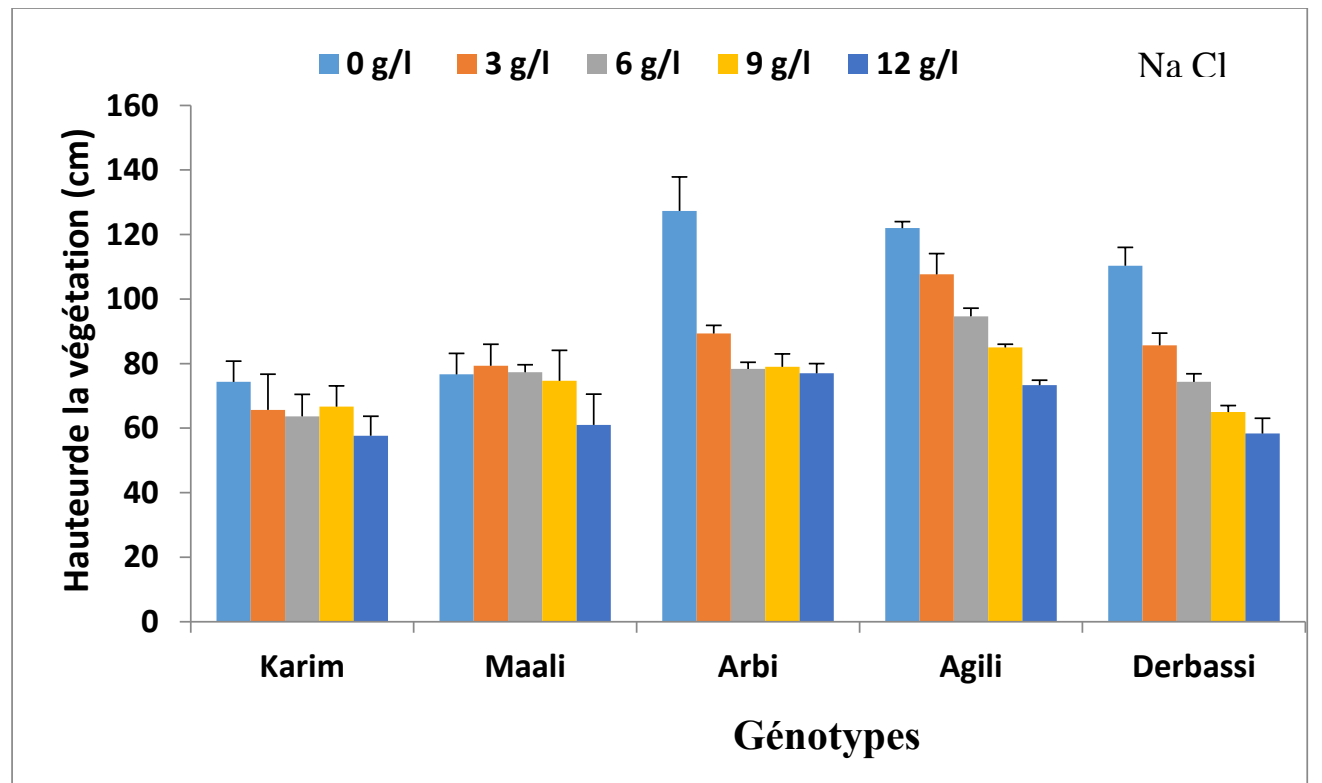

Figure 1: Hauteur de la végétation des différents génotypes étudiés à différents niveaux de stress salin

\section{Nombre de talles par plante}

L'analyse de la variance relative au nombre de talles par plante a montré une différence nette entre les différents niveaux de salinité appliqués ainsi qu'une variation génotypique marquée. Le nombre de talles par plante est un paramètre important qui conditionne le rendement. La figure 2 indique que les génotypes autochtones Agili, Derbassi et Arbi se caractérisent par le nombre de talles le plus important par rapport aux variétés améliorées (Karim et Maali) qui ont montre un nombre de talles très réduit. Il est à noter que cette diminution est relativement proportionnelle à l'augmentation du niveau de sel. En effet, le stress salin implique un raccourcissement du stade tallage et donc une limitation de l'émission de talles dont la conséquence principale est la réduction de la biomasse végétale. Ben Khaled et al. (2007) ont montré que la contrainte saline a pu réduire le nombre de talles même à des doses de 25 et $50 \mathrm{mM} \mathrm{NaCl}$ pour disparaître complètement à partir d'une concentration en $\mathrm{NaCl}$ de $75 \mathrm{mM}$. 


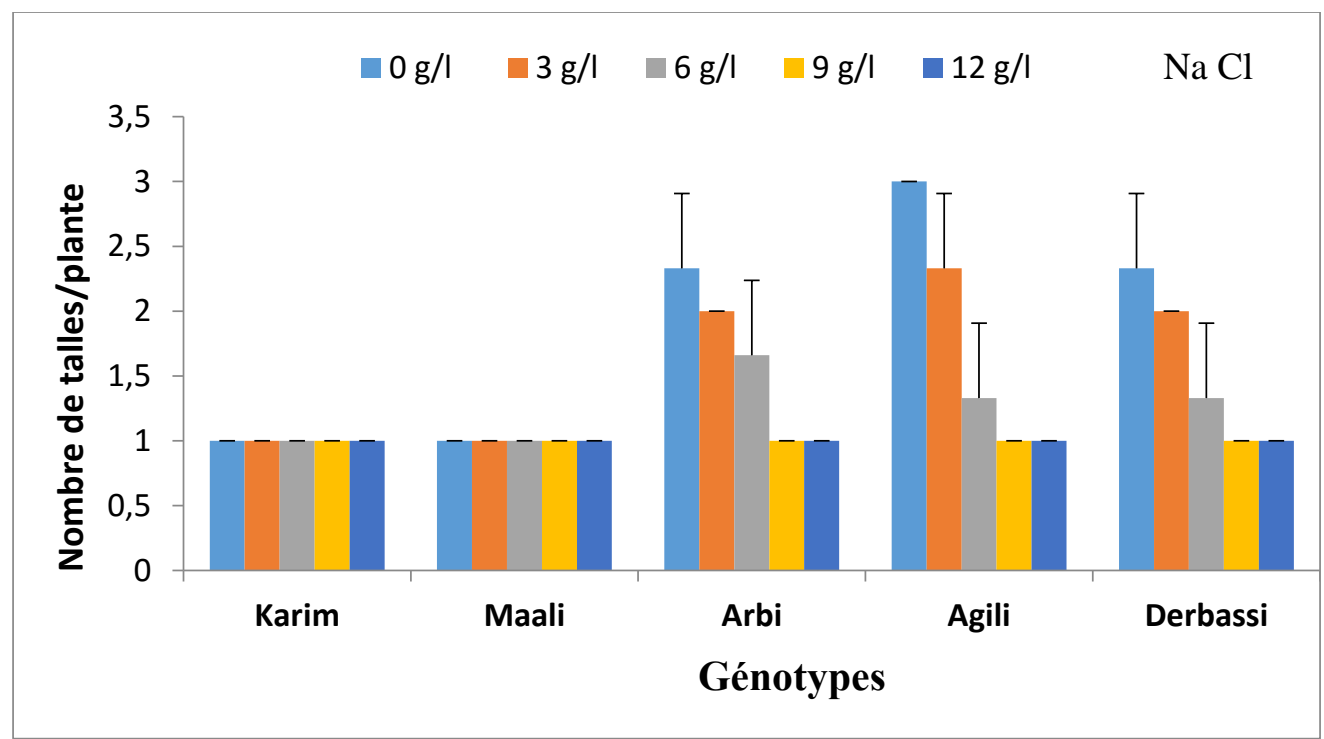

Figure 2: Nombre de talles/plante des différents génotypes étudiés à différents niveaux de stress salin

\section{Nombre de grains par épi}

L'analyse de la figure 3 relative au nombre de grains/épi montre que la salinité réduit significativement ce paramètre. Ce paramètre contribue plus directement au rendement en grain chez le blé dur (Simane et al., 1993). Le nombre des grains par épi des variétés autochtones est plus affecté par l'eau chargée de sel en comparaison avec les variétés améliorées Karim et Maali. Ces résultats montrent que l'augmentation de la salinité de l'eau d'irrigation est associée à une réduction du nombre des grains par épi chez tous les génotypes de blé dur étudiés. En conditions de stress salin, Katerji et al. (1994) et Chamehk et al. (2014) ont prouvé que les paramètres de rendement sont affectés négativement chez le blé dur par la salinité. Les génotypes Derbassi, Arbi et Agili présentent des épis vides pour les traitements 6, 9 et $12 \mathrm{~g} / \mathrm{l}$ de sel. Ce ci pourrait être expliqué par l'effet du stress salin qui affecte la différenciation de l'épi, le nombre d'épillets par épi ainsi que le nombre des grains (Maas et Grieve, 1990; Munns et Rawson, 1999). D'après ElHendawy et al. (2007), la salinité affecte le rendement en réduisant le nombre d'épillets ainsi que leur fertilité. Par conséquent, Maas et Grieve (1990) ont conclu que la salinité doit être évitée avant et pendant le développement de l'épillet pour atteindre le plus haut potentiel de rendement. 


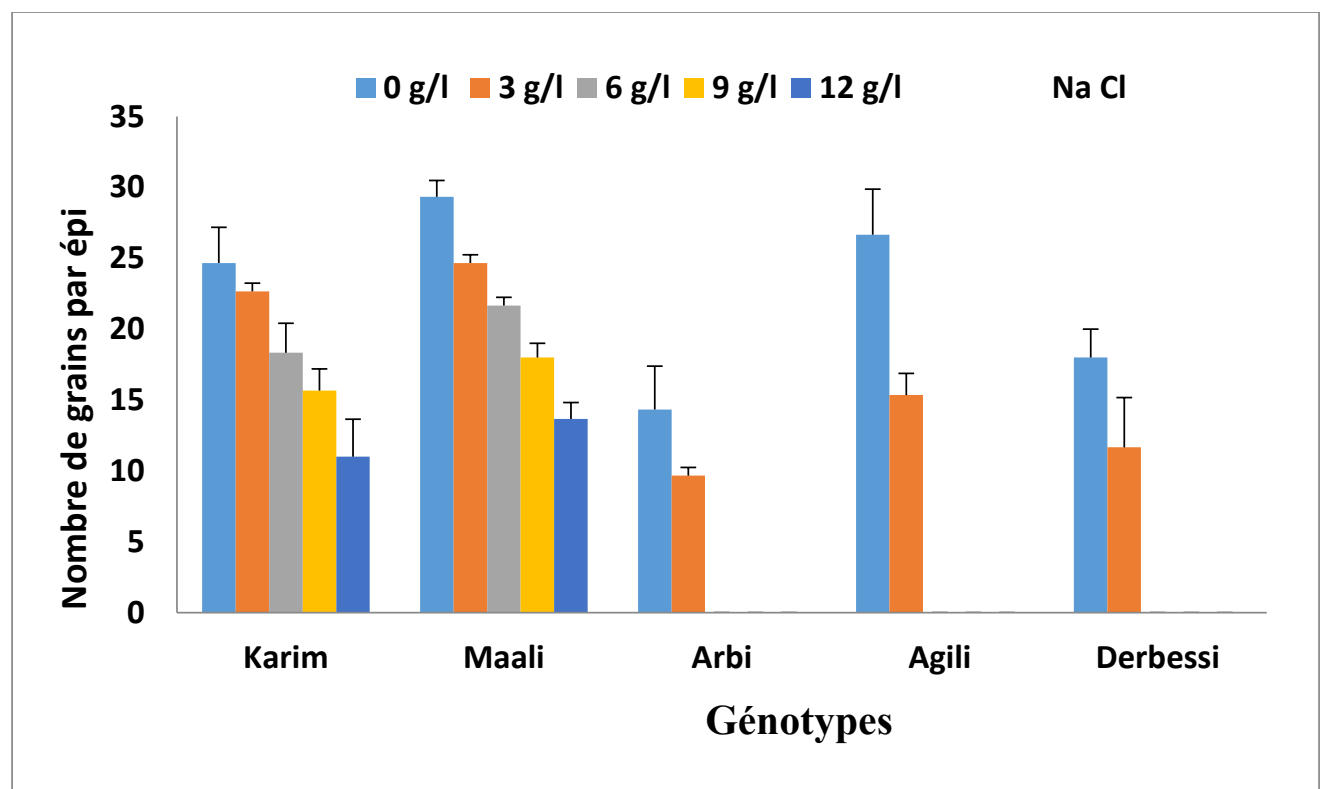

Figure 3 : Nombre de grains/épi des différents génotypes étudiés à différents niveaux de stress salin

\section{Poids de 1000 grains (PMG)}

Les résultats montrent l'effet dépressif du stress salin sur le poids de 1000 grains des différents génotypes de blé dur avec des degrés variables (figure 4), ce qui concorde avec les travaux de Mallek-Maalej et al. (1998). La comparaison des moyennes des différents génotypes a montré que les génotypes Derbassi, Maali et Agili se sont caractérisés par le PMG le plus important. Ce paramètre est peu affecté par la salinité chez les génotypes améliorés Karim et Maali. Lorsque le stress s'intensifie, les différents génotypes ont subit une réduction importante de leurs PMG, ce qui pourrait être expliqué par les problèmes causés par la salinité au niveau de la remobilisation des réserves durant la phase du remplissage. Des résultats similaires ont été obtenus par Francois et al. (1994) et Akram et al. (2002). 


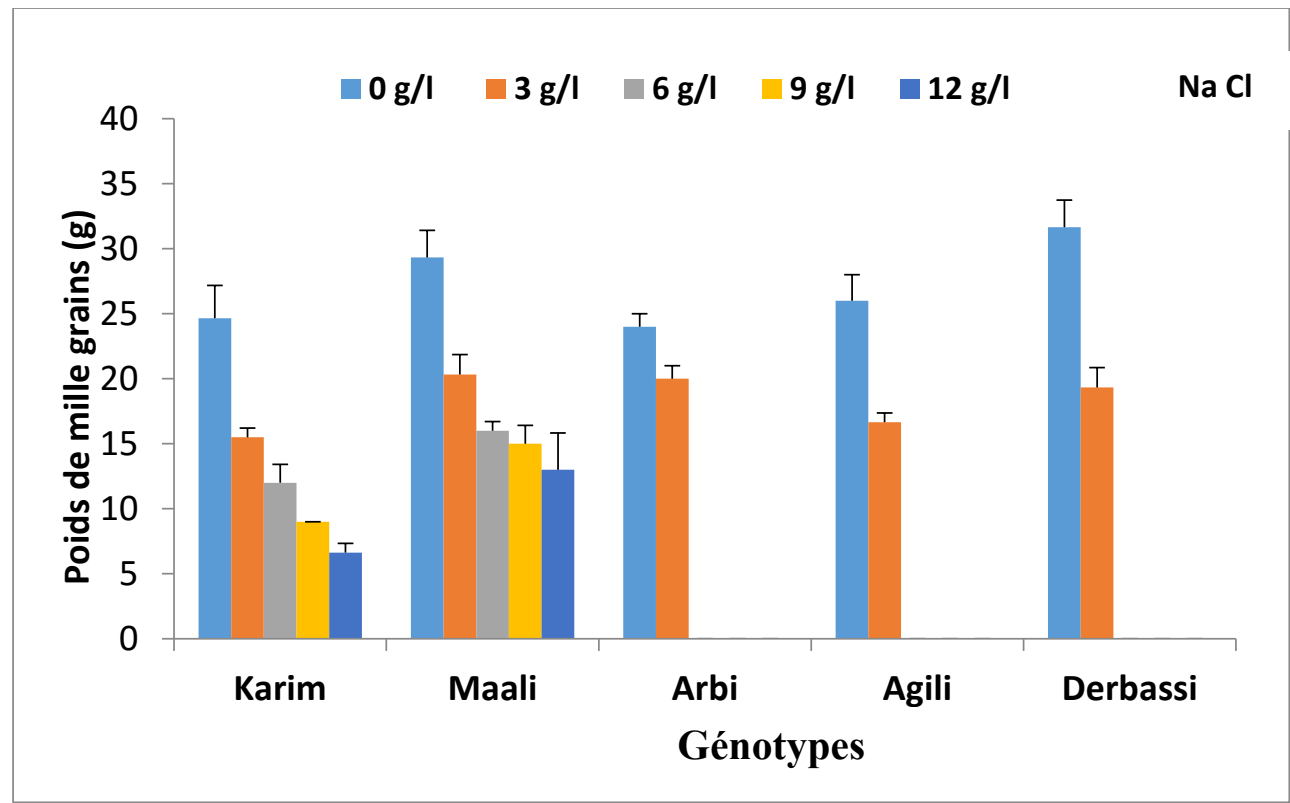

Figure 4: Poids de mille grains des différents génotypes étudiés à différents niveaux de stress salin

\section{Indice de sensibilité relative au sel (I.S.R.S.).}

L'indice de sensibilité relative au sel ou I.S.R.S. montre une importante variation entre les génotypes testés, sous différents niveaux de stress (tableau 2). Lorsque la contrainte est modérée, la variété Karim a révélé l'I.S.R.S le plus important $(1,32)$, alors que les autres génotypes ont présenté les indices assez bas. Sous contrainte sévère $(9 \mathrm{~g} / \mathrm{l}$ et $12 \mathrm{~g} / \mathrm{l})$, des valeurs supérieures à 1 chez tous les génotypes sauf le génotype Maali (0.90). Ce génotype a montré l'Indice de sensibilité relative au sel le plus faible montrant une meilleure adaptation à la contrainte saline en comparaison avec les autres génotypes. Cet indice a été également utilisé par plusieurs auteurs pour différentes espèces pour déterminer la réponse des génotypes d'haricot (Mainassara et al. 2009) et du tabac (Zaghdoud et al. 2011) à la contrainte saline.

Tableau 2: Indice de sensibilité relative au sel (I.S.R.S.) pour tous les génotypes testés sous différents niveaux de sel

\begin{tabular}{|c|c|c|c|c|c|}
\hline \multirow{2}{*}{ Traitements } & \multicolumn{5}{|c|}{ Indice de sensibilité relative au sel } \\
\cline { 2 - 6 } & Agili & Arbi & Derbassi & Karim & Maali \\
\hline Témoin $(0 \mathrm{~g} / 1)$ & - & - & - & - & - \\
\hline $3 \mathrm{~g} / \mathrm{l}$ & 0,81 & 0,77 & 0,73 & 1,32 & 0,63 \\
\hline $6 \mathrm{~g} / \mathrm{l}$ & 0,97 & 0,83 & 0,76 & 1,40 & 0,78 \\
\hline $9 \mathrm{~g} / \mathrm{l}$ & 1,21 & 1,32 & 1,21 & 1,48 & 0,90 \\
\hline $12 \mathrm{~g} / 1$ & 1,40 & 1,63 & 1,38 & 1,60 & 0,92 \\
\hline
\end{tabular}


Les résultats de la culture en pot obtenus révèlent que les génotypes Maali, Derbessi et Agili ont montrés les meilleurs résultats en terme de nombre de talles par plante, nombre d'épis par plante ainsi que les faibles I.S.R.S. Cependant, ces génotypes ont présenté des épis vides sous stress salin $(6,9$ et $12 \mathrm{~g} / \mathrm{l})$ comparativement à la variété améliorée Karim. La variété Maali s'est distinguée par une tolérance à la salinité en maintenant un poids de mille grains important comparativement aux témoins non stressés. Par conséquent, il est intéressant de tester cette variété sur champ en sols salins pour confirmer les résultats que nous avons obtenus durant les deux essais en phase de germination (Ayed et al., 2014) et en culture en pot.

\section{Conclusion}

Cette étude, qui mérite d'être approfondie, permet de conclure que les caractères agro-morphologiques (nombre de talles par plante, hauteur de la végétation, nombre de grains par épi et poids de mille grains), pris séparément, ne peuvent permettre la sélection de génotypes tolérants à la salinité chez le blé dur. Toutefois, leur combinaison peut constituer la base de cette sélection. Ainsi, un génotype tolérant devrait montrer une performance agronomique qui pourrait être confirmée par une étude physiologique pour confirmer ces résultats.

\section{References:}

Al Karaki G.N. \& R.B. Clark, 1998. Growth, mineral acquisition, and water use by mycorrhizal wheat grown under water stress. J. Plant Nutr., 21, 263267

Akram M, Hussain M., Akhtar S., Rasul E., 2002. Impact of $\mathrm{NaCl}$ salinity on yield components of some wheat accessions/varieties. Lnt. J. Agric. Biol., 1: 156-8

Asgaria H.R., Cornelisb W., Van Dammec P. (2012) Salt stress effect on wheat (Triticum aestivum L.) growth and leaf ion concentrations. International Journal of Plant Production (2): 195- 208

Ayed S., Rassa N., Chamek Z., Beji S., Karoui F., Bouzein T., Mrabit M. and Ben Younes M., 2014. Effect of salt stress (sodium chloride) on germination and seedling growth of durum wheat (Triticum durum Desf.) genotypes. International Journal of Biodiversity and Conservation Vol. 6 (4): 320-325.

Ben Naceur M., Rahmoune Ch., Sdiri H., Meddahi M.L. et Selmi M., 2001. Effet du stress salin sur la germination, la croissance et la production en grains de quelques variétés maghrébines de blé. Sécheresse $2001 ; 12$; pp : 167-174. 
Ben Khaled L., Ouarraqi E.M. et Zid E., 2007. Impact du $\mathrm{NaCl}$ sur la croissance et la nutrition de la variété de blé dur Massa cultivée en milieu hydroponique. Acta Botanica Gallica, 154 (1):101-116.

Chamekh Z., Ayed S., Sahli A., Ayadi S., Hammami Z., Jallouli S., Trifa Y. and Amara H. (2014). Effects of salt stress on the flag leaf area, ion accumulation and components yield of durum wheat genotypes (Triticum turgidum ssp durum) in Tunisia. Journal of New Sciences Vol. 6(3), 15-23.

Cheraghi S.A.M., 2004. Institutional and scientific profiles of organizations working on saline agriculture in Iran. In: Prospects of Saline Agriculture in the Arabian Peninsula: Proceedings of the International Seminar on Prospects of Saline Agriculture in the GCC Countries. (Ed.), Taha, F.K., Ismail, S., Jaradat, A. 18-20 March 2001, Dubai, United Arab Emirates, pp. 399-412.

Cordovilla M.P., Ocana A., Ligero F. \& Lluch C., 1995b. Growth stage response to salinity in symbiosis Vicia faba-Rhizobium leguminosarum bv. viciae.Plant Physiol., 14, 105-111.

Deghaïs M., Kouki M., Gharbi M.S. et El Felah M., 2007. Les variétés de céréales cultivées en Tunisie.2007. 445p.

Dehdari A., Rezai A., Maibody S.A.M., 2007. Genetic control of salt tolerance in wheat plants using generation means and variances analysis. $\mathrm{J}$. Sci. and Technol. Agric. and Natur. Resour. 11, 40-49.

Delgado M.J., Ligero F. \& Lluch C., 1994. Effects of salt stress on growth and nitrogen fixation by pea, faba-bean, common bean and soybean plants. Soil Biol. Biochem., 26, 371-376.

El-hendawy S.E., Hu Y., Yakout G.M., Awad A.M., Hafiz S.E. et Schmidhalter U., 2007. Evaluating salt tolerance of wheat genotypes using multiple parameters. European Journal of Agronomy. 22. pp: 243-253.

FAO, 2013. An international vision for wheat improvement

Francois LE., Grieve CM., Maas EV., Lesch SM., 1994. Time of salt stress affects growth and yield components of irrigated wheat. Agron. J., 86: 100107.

Fisher R.A. \& Maurer R., 1978. Drought resistance in spring wheat cultivars. I. Grain yield responses. Aust. J. Agric. Res., 29, 897-912.

Fricke W., Peters W.S., 2002. The biophysics of leaf growth in salt-stressed barley: a study at the cell level. Plant Physiol. 129:374-388

Gholamin R., Zaeifizadeh M., Khayatnezhad M., 2010. Factor Analysis for Performance and Other Characteristics in Durum Wheat under Drought Stress and Without Stress. Middle-East Journal of Scientific Research 6 (6): 599-603

Hameed M., Naz N., Ahmad M.S.A., Islam-ud-Din, Riaz A., 2008. Morphological adaptations of some grasses from the salt range, Pakistan. Pak. J. Bot., 40: 1571-1578. 
Hemati R., Pakniyat H., 2006. Evaluation of bread wheat (Triticum aestivum L.) and durum wheat genotypes under salinity stress. Iran J. Agric. Sci., 37: 239-49

Katerji N., Van Horn J.W., Hamdy A., Karam F., Mastrorilli M., 1994. Effect of salinity on emergence and on water stress and early seedling growth of sunflower and maize. Agric. Water Mang. 26:81-91.

Katerji N., Mastrorilli M., Rana G., 2006. Analysis and improvement of water use efficiency for crops cultivated in the Mediterranean regions: the state of the art. WEMED Project Report, CIHEAM Bari, Italy, 32 p.

Läuchli A. and Epstein E.. 1990. Plant responses to saline and sodic conditions. In K.K. Tanji (ed). Agricultural salinity assessment and management. ASCE manuals and reports on engineering practice No, 71. pp 113-137 ASCE New York

Levigneron A., Lopez F., Vansuyt G., 1995. Les plantes face au stress salin. Cahiers Agricultures 4: 263-273.

Maas E.V., Grieve C.M.. 1990. Spike and leaf development in salt-stressed wheat. Crop Sci. 30:1309-1313.

Maalej-Mallek E., Boulesnam F., Ben Salem M., 1998. Effet de la salinité sur la germination de graines de céréales cultivées en Tunisie. Cahiers Agricultures. 2: 153-6.

Mainassara Z, Sifi B., L' TAIEF B., El Aouni M. H., 2009. Paramètres agronomiques liés à la tolérance au sel chez le haricot (Phaseolus vulgaris L.). Biotechnologie, Agronomie, Société et Environnement (13) : 113-119

Munns R., Tester M., 2008. Mechanisms of salinity Tolerance. Annual Review of Plant Biology, Vol.59; pp: 651-681.

Munns R., 2002. Comparative physiology of salt and water stress. Plant Cell Environ., 25, 239-250.

Munns, R. and Rawson H.M., 1999. Effect of salinity on salt accumulation and reproductive development in the apical meristem of wheat and barley. Aust. J. Plant Physiol. 26:459-464.

Niu X., R.A. Bressan, Hasegawa P.M., Pardo J.M., 1995. Ion homeostasis in $\mathrm{NaCl}$ stress environments. Plant Physiol., 109, 735-742.

Parida A.K., Das A.B., 2005. Salt tolerance and salinity effects on plants: A.Rev.Ecotoxicol.nviron. Safety, 60: 324-349.

Simane B., Peacock J.M., Strick P.C., 1993. Differences in development plasticity growth rate among drought . resistant and susceptible cultivars of durum wheat ( Triticum turgidum L.Var. durum) . Plant and soil, 157, 155166.

Shannon M.C., 1997. Adaptation of plants to salinity. Adv. Agron., 60, 75120. 
Zaghdoud C., Debouba M., Maâroufi-Dguimi H., Gouia H., 2011. Comportement physiologique de deux espèces de tabac au stress salin 'Nicotiana tabacum' et 'Nicotiana rustica'. Revue des Régions Arides (25): 3-14. 